\title{
Improving, and auditing, access to clinical trial results
}

Part of the third sentence in the penultimate paragraph of this Editorial (BMJ 2014;348:g213, doi:10.1136/bmj.g213) was cut by the $B M J$ to save space in error. The third sentence should have read: "Evidence from the Cochrane Collaboration seems to show that this is not true" rather than "Evidence from the Cochrane Collaboration shows that this is not true."

Cite this as: BMJ 2014;348:g276

๑ BMJ Publishing Group Ltd 2014 